\title{
1 The Wessex Dementia Friendly Pharmacy Framework
}

2

3 Amanda Moores ${ }^{1}$ and Paul Rutter ${ }^{2}$

4

$5{ }^{1}$ Dorset Local Pharmaceutical Committee, Merley House, Wimborne, UK

$6{ }^{2}$ School of Pharmacy and Biomedical Sciences, Portsmouth University, Portsmouth, 7 UK

8

9 
11 Abstract

12 Objectives

13 To develop and launch a dementia friendly framework for community pharmacies in

14 the Wessex region of England.

Methods

A framework consisting of essential (mandatory) and additional (non-mandatory) criteria were devised by local stakeholders and external scrutiny from the Alzheimer's Society. The framework was designed to allow pharmacy teams to achieve essential criteria without the need for approval by others (e.g. authorisation from internal company management structures). In total 38 essential criteria across seven domains were devised. All essential criteria had to be met for pharmacies to be awarded dementia friendly status. Engagement events were organised to launch the framework detailing what it was and how pharmacies could meet each criterion. Pharmacies self-certified compliance with the framework criteria via an online platform, and validation activity was subsequently undertaken to see if pharmacies had appropriately self-certified against all essential criteria.

Key findings

355 pharmacies $(n=504,70 \%)$ engaged with the initiative, of which 330 uploaded self-certifications met all essential criteria of the framework. Validation visits showed self-certification to be appropriate in all but one visit $(n=11 / 12)$. Staff comments revealed that engaging with the framework had allowed them to be more aware of how better to support people with dementia. 
35 A dementia friendly framework was devised and implemented with a subsequent

36 high level of uptake by community pharmacy.

37

38

Keywords

39 Community pharmacy services; dementia; community pharmacists

40

41

42

43

44

45

46

47

48

49

50

51

52

53 
Introduction

Almost one million people currently live with dementia in the UK, with this set to rise to over two million by $2050 .{ }^{1}$ The annual economic burden is $£ 26$ billion a year, and is predicted to rise to $£ 59.4$ billion by $2050 .^{2}$ As dementia cannot be cured, care is centred on helping to make their lives easier by providing support, for example adapting environments to their needs, access to carers and managing medication. Community pharmacists' have regular contact with dementia patients/their carers affording them opportunities to provide support. However, the type and level of support offered is unknown despite recent (2017) financial incentivisation through the nationally negotiated English Community Pharmacy Contractual Framework to ensure that $80 \%$ of pharmacy staff in patient facing roles were recognised as 'dementia friends'3 as detailed by fulfilling the Alzheimer's Society toolkit, which seeks to change peoples' attitudes and further peoples understanding of dementia through provision of taught materials and watching videos on dementia. By becoming a dementia friend, pharmacy team members can then understand the patient's needs and raises awareness of the stigma and common misconceptions surrounding dementia.

In Wessex (counties of Hampshire, Dorset and the Isle of Wight on the south coast of England) uptake was almost universal $(96 \%, n=483 / 504)$, and consequently local National Health Service stakeholders, the Wessex Pharmacy Local Professional Network (LPN) and commissioners wanted to capitalise on this level of engagement by developing a framework to enable pharmacies to become "Dementia Friendly". To 
support this initiative funding was secured from NHS England primary care transformational funding.

This service innovation aimed to develop and roll out a dementia friendly framework to community pharmacies and to determine uptake and adherence with the

81 framework.

Methods

A working group, comprising representatives from NHSE Wessex, chairs of the local pharmacy network, Public Health Dorset and both local pharmaceutical committees within the Wessex geography met 3 times to establish the framework. Previous work in Greater Manchester, England was used as a starting point in devising the Wessex Dementia Friendly Framework, namely adoption of seven broad domains encompassing delivery of pharmacy services. ${ }^{4}$ In addition, reference to the Wessex Ispace project, which looked to make family doctor practices more dementia friendly was consulted.

\section{(https://wessexahsn.org.uk/img/news/Dementia\%20Friendly\%20Surgeries\%20Sum} mary\%20Report\%202017.pdf)

Under each of the seven domains both essential (mandatory) and additional (nonmandatory) criteria were incorporated and drew on previous published guidance. .-7 $^{5-1}$

The criteria were grouped under headings to provide a logical and practical

97 framework for pharmacy teams that covered most aspects of the pharmacy as a whole. The framework was designed to be achievable with a pharmacy team working together to deliver the different aspects. 
Essential criteria were deemed things that were achievable by the pharmacy team without the need for approval by others (e.g. activities within the team's control and not requiring authorisation from internal company management structures). In total 38 essential criteria across the seven domains were devised. All essential criteria had to be met for pharmacies to be awarded dementia friendly status. There was no such mandatory requirement for those listed as additional criteria. Prior to the launch of the agreed framework it was sent for external comment to Alzheimer's Society. No structural changes were made to the framework as a result of this consultation.

All community pharmacies $(n=504)$ within NHSE Wessex were written to in December 2017 and were invited to attend one of nine engagement events. The letter contained the information of how to register for an event, detailed the topics under discussion and outlined what payments would be made for successful completion of the framework. Engagement events were held in January 2018 to launch the framework. A total of 346 people representing 238 pharmacies attended. The events covered the background to why the project was being undertaken; key facts about dementia; why being dementia friendly is important; and Connie's story, which followed the life of Connie who had dementia and the positive impact a pharmacy team could have supporting a person with dementia. The events also included the mechanism by which self-certification against the framework would be captured via an on-line platform provided by Pharmoutcomes (web-based IT system allowing data capture of pharmacy services to allow commissioners to audit their activity). Staff were asked to log on to this system where the framework could be 
accessed to select yes/no against each of the criteria. The responses were summarised to show overall compliant (green) or non-compliant (red) status for the pharmacy. Each pharmacy could keep accessing the platform up until the deadline to update their responses, and amend their status as relevant.

As it was not compulsory to attend an engagement event, the two LPCs also sent out information directly to all pharmacies to encourage uptake.

Pharmacies had until $31^{\text {st }}$ March 2018 to upload self-certification. If all 38 essential criteria were completed through this process then pharmacies were awarded a $£ 400$ payment. As part of the service specification for funding, a validation process was put in place where purposive selected pharmacies were visited 2 to 3 months after the March deadline to check that self-certification was accurate. Both independent pharmacy contractors and pharmacies part of a chain were included. The visits were undertaken by two members of a group of three who had delivered the engagement events. AM was involved in 8 of the 12 visits. A template validation visit report was developed and included specific information to see/ask about for each criteria within the framework. In addition three further questions were asked exploring engagement with the additional criteria, how the framework had altered pharmacy operation and what had been learnt from the process. Answers to these questions were directly recorded on to the template. No audio recordings were made. Data was thematically analysed by PR. If any pharmacy failed this validation visit dementia friendly status and any payments were withdrawn.

There was no requirement for ethical approval as this was an extension to the service already offered by community pharmacies and did not constitute research as defined by NHS health research authority. However, all staff from the pharmacies 
148 visited did give verbal consent for their data to be used in published materials.

149 Nobody approached to be interviewed refused this request.

151 Results

152

Data were received from 355 (70\%) pharmacies, of which 324 self-certified that they had met all 38 essential criteria. Of the 31 that did not, 19 pharmacies achieved scores between 35 and 37; these pharmacies, along with a further 5 pharmacies that had technical issues of uploading data to the online platform, were given an additional 7 days to review their entries. As a result 330 pharmacies across Wessex were accepted and given Dementia Friendly Pharmacy status.

Forty-five staff members from the 12 pharmacies (range 3-7 staff per pharmacy) were spoken to during the visits to establish compliance with the framework. Those spoken to included pharmacists, accredited checking technicians, technicians/dispensers, medicine counter assistants, health champion and managers. The validation process showed that 11 of the 12 pharmacies visited did demonstrate compliance with all 38 essential criteria. Only one pharmacy was deemed not to fully comply, as staff were unable to answer all questions about the framework or show documented information to support their claim of meeting the essential criteria. This pharmacy was the only one whose staff had not attended an engagement event. Apart from this one pharmacy, it was apparent that the framework had made staff more aware of how better to support patients with dementia as exemplified by comments such as: 
172 'Awareness amongst the staff has greatly increased in terms of factors such as the environment and colours...... The pharmacy team have been sharing details with colleagues in other areas of the store e.g. opticians, check out and also with their line manager and above. [multiple pharmacy chain located in a superstore]

'We were already doing a lot of things, however the framework has helped formalise the process and make thinks more official.....It has certainly raised awareness of dementia amongst the pharmacy team. It has also helped with handling/dealing with queries from patients with dementia and in particular with any patient who is about to start having additional support e.g. medication compliance aids. The team will now take patients with dementia to a quiet area or to the consultation room away from the counter when serving them.... This [the framework] has made the staff more aware of what they can do to support patients who may have memory issues [independent pharmacy chain].

'The staff have more confidence about giving advice on how to prevent dementia. By doing the framework the team members have realised how much they can support each other as well as their patients as it has allowed members of the team to openly discuss how someone in their family having dementia has impacted on their own personal lives' [multiple pharmacy chain located in a superstore]. 
A dementia friendly framework was developed and implemented across a large number of pharmacies in the South of England. It showed that with incentivisation pharmacies were willing to become Dementia Friendly. However, validation visits did suggest that engagement was more than just monetary as staff were able to provide examples of changes in their practice and felt more empowered to talk with patients.

This seems to suggest the framework is a useful vehicle by which community pharmacies can improve the way they provide services to patients with dementia as advocated by previous research. ${ }^{8-9}$

The success of this innovation utilising a framework may have partially been influenced by English community pharmacies familiarity with such approaches - for example the adoption of the now national Healthy Living Pharmacy concept. ${ }^{10}$ Such framework approaches to augment innovation in community pharmacy are generic healthcare structures to achieve improvements in patient care. Ideally such approaches need to be integrated across health and social care to provide coordinated care pathways for patients - something which was lacking in this service innovation as exemplified by medical practices and community pharmacies independently creating solutions to be more dementia friendly.

We do recognise that these findings must be tempered by the nature of selfcertification and the small proportion of validation visits. However, this type of

217 initiative appears to have been recognised by NHS England as the new contractual framework for community pharmacy for $2019 / 20$ to $2023 / 24$, includes the need for all patient facing staff to be Dementia Friends and for the pharmacy to complete a 
"specified dementia-friendly environment checklist and action plan". Whilst this was not identical to the framework used in Wessex there were similarities and it is

222 possible the successful work completed within Wessex influenced the national

223 thinking around dementia.

224 Conclusion

225 Co-operation between stakeholders, commissioners and community pharmacy

226 teams allowed the dementia friendly framework to be devised and implemented with

227 a high level of uptake by community pharmacy. 
1. Alzheimer's Society (2014.). Opportunity for change.

https://www.alzheimers.org.uk/sites/default/files/migrate/downloads/dementia 2014 opportunity for change.pdf. (accessed September 23, 2020).

2. Community Pharmacy South Central (2019). Dementia Friendly Pharmacies. https://www.cpsc.org.uk/professionals/dementia-friendly-pharmacies. (accessed 250 September 23, 2020).

3. Pharmaceutical Services Negotiating Committee Briefing 054/18 (2018) Quality Payments - How to become a Dementia Friend. Pharmaceutical Services Negotiating Committee. https://psnc.org.uk/wp-content/uploads/2018/10/PSNCBriefing-054.18-Quality-Payments-2018-19-How-to-become-a-Dementia-Friend.pdf 256 (accessed 4 March, 2020).

4. Greater Manchester LPC. (2019). Dementia-Friendly Pharmacy. https://psnc.org.uk/greater-manchester-Ipc/service-information/dementia-friendly-

259 pharmacy-framework/ (accessed 4 March, 2020). guide for employers. https://www.alzheimers.org.uk/sites/default/files/migrate/downloads/creating_a_dem entia-friendly_workplace.pdf (accessed 6 May, 2020). 
6. Alzheimer's Australia (2017). Creating dementia-friendly communities. Business toolkit. https://www.dementiafriendly.org.au/sites/default/files/resources/The-

\section{7} Dementia-friendly Business-Toolkit.pdf (accessed 6 May, 2020).

7. Stafford A. The pharmacist's role in supporting people living with dementia in the community. Australian Pharmacist. 2015:36;36-39.

8. Bennett KA. Supporting pharmacy practice: Creating a dementia-friendly 272 pharmacy. Australian Pharmacist. 2015;34:50-52. engagement and consultation to inform the development of ageing- and dementia friendly pharmacies - Innovative practice. Dementia. 2017; 4:1237-1243. https://doi.org/10.1177/1471301217725896. Pharmacies. https://psnc.org.uk/services-commissioning/locally-commissionedservices/healthy-living-pharmacies/ (accessed 23 September 2020). 